\title{
Off-limb EUV line profiles and the search for wave activity in the low corona
}

\author{
R. A. Harrison ${ }^{1}$, A. W. Hood ${ }^{2}$, and C. D. Pike ${ }^{3}$ \\ ${ }^{1}$ Space Science and Technology Dept., Rutherford Appleton Laboratory, Chilton, Didcot, Oxfordshire OX11 0QX, UK \\ and Honorary Visiting Professor, School of Mathematics and Statistics, the University of St Andrews \\ 2 School of Mathematics and Statistics, The University of St Andrews, St Andrews, Fife KY16 9SS, Scotland, UK \\ 3 Space Science and Technology Dept., Rutherford Appleton Laboratory, Chilton, Didcot, Oxfordshire OX11 0QX, UK
}

Received 30 April 2002 / Accepted 21 June 2002

\begin{abstract}
Two extreme-ultraviolet (EUV) observations are used to examine the off-limb characteristics of emission line widths of the million K Mg X $625 \AA$ line. To obtain sufficient statistical accuracy, the observations were made over long periods of time and a pixel summing technique is used. The observations are made above the western limb, for quiet corona. The most significant result is the discovery of emission line narrowing as a function of altitude and intensity at altitudes above $50000 \mathrm{~km}$. The results are compared to past observations, which suggest that emission line broadening, at lower altitudes, is due to the outward propagation of undamped Alfvén waves, in open field regions with decreasing density with altitude. The narrowing at higher altitudes, determined by the current study is interpreted as further evidence for coronal wave activity, but in closed field regions, and most likely the first evidence of the dissipation of Alfvén waves in the corona. An additional result of this work is the identification of a significant line broadening across a narrow region on the limb, which may be due to flows in low-lying loop systems.
\end{abstract}

Key words. Sun: UV radiation - Sun: atmosphere - Sun: corona

\section{Introduction}

In the quest to understand the fundamental processes driving the heating of the solar corona and acceleration of the solar wind, many researchers have pointed to the potential role of wave activity. Such waves would be driven ultimately by the convective motion within the body of the Sun. Of course, the ultimate test for any theoretical heating and/or acceleration model based on wave activity is the detection of wave activity in the first place, and evidence for waves in the solar atmosphere is scarce.

Hassler et al. (1990) made an early attempt at determining the off-limb line-width profile of coronal emission lines using a rocket-borne instrument. Using the million K Mg X 609 and $625 \AA$ line data they identified what appeared to be an increasing line-width with altitude above the limb up to altitudes of $70000 \mathrm{~km}$, above which the line-width appeared to become constant to an altitude of $140000 \mathrm{~km}$. The line-width increase was interpreted as the signature of outward propagating undamped Alfvén waves. The observation was a one-off measurement with spatial pixels of $20 \operatorname{arcsec} \times 60 \operatorname{arcsec}$ and the nature of the limb region being examined (i.e. quiet Sun,

Send offprint requests to: R. A. Harrison,

e-mail: r.harrison@rl.ac.uk coronal hole or active region components) is unclear. However, the results gave a strong indication that Alfvén wave activity could be identified off the limb.

More recently, there have been a number of off-limb studies made using instrumentation on board the SOHO spacecraft. For example, Doyle et al. (1998) and Banerjee et al. (1998) have studied off-limb line-width characteristics using the $8 \times 10^{5} \mathrm{~K} \mathrm{Si}$ VIII lines at $1440.49 \AA$ and $1445.75 \AA$ A Doyle et al. made observations to an altitude of $25000 \mathrm{~km}$ above the equatorial limb, and identified a broadening of emission lines from an effective non-thermal velocity broadening of $24 \mathrm{~km} \mathrm{~s}^{-1}$ to $28 \mathrm{~km} \mathrm{~s}^{-1}$ along with a density decrease. Similarly, above a coronal hole, Banarjee et al. made observations to an altitude of $180000 \mathrm{~km}$ and identified a broadening with altitude up to $110000 \mathrm{~km}$ (150 arcsec off-limb). Their data suggested a nonthermal broadening from $28 \mathrm{~km} \mathrm{~s}^{-1}$ at the limb to $45 \mathrm{~km} \mathrm{~s}^{-1}$ at $110000 \mathrm{~km}$, with an associated density decrease. Both the Doyle et al. and Banerjee et al. results are consistent with the outward propagation of undamped Alfvén waves travelling through regions of decreasing density (see Moran 2001).

Doyle et al. (1999) extended their own work to altitudes of $250000 \mathrm{~km}$, in a coronal hole. In this case, they demonstrated that the Si VIII line showed a line width increase to an altitude of about $100000 \mathrm{~km}$. Above this there was evidence for the 
width levelling off, and there was a hint of a fall-off in the width above $220000 \mathrm{~km}$. However, their uncertainties were too great to show that this was a real effect. Their data were also plotted in Fig. 10 of Banerjee et al. (2000). However, the Banerjee et al. study also showed a continuous increase in the O VI line width with altitude up to about $280000 \mathrm{~km}$.

Doschek et al. (2001) studied several emission lines, showing increasing widths with altitude, again for a coronal hole. Above an altitude of about $75000-100000 \mathrm{~km}$, there was a hint of flattening in the width-altitude curves, but the significance of this was in question because of the large uncertainty in the data. Doschek \& Feldman (2000) extended this to a study above the equator. Their Table 1 listed line widths for 7 emission lines from ions in the range 300000 to $2000000 \mathrm{~K}$, for altitudes up to 0.45 solar radii above the limb. They concluded that with a net uncertainty of $30 \%$, they could not justify any specific conclusion about the line width-altitude profile.

These analyses beg further inspection of the emission linewidth characteristics off the limb, and, in this paper, we present results of an analyis of extreme-ultraviolet (EUV) line widths in the equatorial corona up to an altitude of $150000 \mathrm{~km}$ using principally the million K Mg X $624.94 \AA$ line. This approach is similar to the Hassler et al. (1990) study, but with considerably improved spatial resolution over a much longer period of time, with a very quiet corona, and is complementary to the Doyle et al. (1998) study, which was also on the equatorial limb, but to an altitude 6 times greater.

The study makes use of two dedicated, unique sets of longduration off-limb observations of the quiet corona above the western limb. These data allow an examination of the relationship between coronal emission line profiles as a function of altitude and intensity to an altitude of $150000 \mathrm{~km}$. In particular, we identify coronal emission line narrowing with altitude above $50000 \mathrm{~km}$. Unlike the previous analyses, we believe that the interpretation of the data supports not just the propagation of Alfvén waves into closed loop features, but suggests that we are witnessing wave dissipation. We discuss two basic theoretical interpretations of the narrowing, namely through the damping of Alfvén waves in closed loops due to resistivity or viscosity, and the phase-mixing of Alfvén waves.

In the next section we outline the observation scheme and provide details of the instrumentation. This is followed by an analysis of the specific data-sets in question. Results from this are then compared to a theoretical interpretation in the subsequent section.

\section{The EUV observation scheme and analysis}

We make use of observations from the Coronal Diagnostic Spectrometer (CDS; Harrison et al. 1995), aboard the European Space Agency/NASA Solar and Heliospheric Observatory (SOHO). CDS is an EUV spectrometer operating in the range from 150 to $800 \AA$. It allows the detection and analysis of spectral emission lines from numerous trace ions in the solar atmosphere, with spatial, spectral and temporal resolving elements of down to 2 arcsec, $0.08 \AA$, and a few seconds respectively (see Harrison et al. 1995 for a full description). The CDS wavelength region contains emission lines which come from ions with characteristic temperatures in the range $20000 \mathrm{~K}$ to over 2 million $\mathrm{K}$, thus making this particular wavelength region extremely valuable for studies of the solar atmosphere, where large temperature ranges exist.

We report on two dedicated long-duration observations, which were made of the western solar limb using the Nornal Incidence Spectrometer (NIS) component of the CDS instrument. For each, a 4 arcsec $\times 240$ arcsec slit was rastered over a $240 \operatorname{arcsec} \times 240$ arcsec field of view, and spectral data for each exposure of the raster were returned for four selected wavelength bands, centred on O V $629 \AA$ (250000 K), Mg X $624 \AA$, Mg IX $368 \AA$ (both 1 million K) and Fe XVI $360 \AA$ ( 2 million $\mathrm{K}$ ). The basic aim was to return data from a good range of temperatures. The exposure time at each of the 60 rastered positions was $30 \mathrm{~s}$. The NIS is stigmatic, that is, we have a spatial focus along the slit, with spatial resolution 1.68 arcsec (143 pixels along the 240 arcsec slit). Some 40 (spectral) pixels were returned across each of the chosen wavelength bands thus allowing good knowledge of the emission line profiles and the background intensity levels. Each spectral pixel for the $\mathrm{O} \mathrm{V}$ and $\mathrm{Mg} \mathrm{X}$ data represents $0.14 \AA$ (a band-width of $3.2 \AA$ ), whilst for the Mg IX and Fe XVI data, the pixels represent $0.08 \AA$ (a band-width of $5.6 \AA$ ). In this analysis we are attempting to identify potentially weak line broadening and thus have to consider any influences from line blends in particular. The $\mathrm{O} \mathrm{V}$ spectral window is dominated by the bright O V $629.73 \AA$ emission line. In the current analysis, we use this line to identify principally the limb position; its intensity falls effectively to zero off-limb. The Mg IX window is dominated by the Mg IX $368.07 \AA$ line, but, significant, yet weaker Mg VII lines are found at 367.67 and $367.68 \AA$. $\mathrm{Mg}$ VII has a characteristic temperature of $6.3 \times 10^{5} \mathrm{~K}$. This blend means that temperature changes with altitude could influence any line width measurement as the ratio of the Mg IX and $\mathrm{Mg}$ VII line intensities vary. For the $\mathrm{Mg} \mathrm{X}$ window, the principal line is the $\mathrm{Mg} \mathrm{X} 624.94 \AA$ line. Weak O IV lines exist at 624.62 and $625.13 \AA$. However, these have a characteristic temperature of $1.6 \times 10^{5} \mathrm{~K}$. The fact that the $2.5 \times 10^{5} \mathrm{~K} \mathrm{O} \mathrm{V}$ emission line intensity falls to zero off the limb would suggest that any blend with the O IV data, well off-limb is not of concern. A weak Si X line is also found at $624.73 \AA$. The Si X ion has a similar temperature to the $\mathrm{Mg} \mathrm{X}$ line. Thus, we do not expect the ratio of $\mathrm{Si} \mathrm{X}$ to $\mathrm{Mg} \mathrm{X}$ intensities to change with altitude/temperature. Finally, the Fe XVI window is dominated by the Fe XVI $360.76 \AA$ line, but also includes a strong Fe XIII line at $359.84 \AA$. Again, variations in temperature will influence any line profile fits of the Fe XVI data.

The presence of blends is a fact of life in the crowded EUV emission line spectrum. Whilst the Mg IX and Fe XVI data will be analysed as part of this investigation, analysis of the line-width of the $\mathrm{Mg} \mathrm{X}$ line is clearly less influenced by the presence of blends and we present the $\mathrm{Mg} \mathrm{X}$ analysis as a first report on this work.

In Fig. 1, we show two rasters from the datasets, taken on July 5 and July 8, 2001. Images produced from the $\mathrm{O}$ V and $\mathrm{Mg} \mathrm{X}$ data are shown.

For the July 5, 2001 data, observations were made from 03:17 UT and 12 rasters were made, ending at 10:04 UT. 

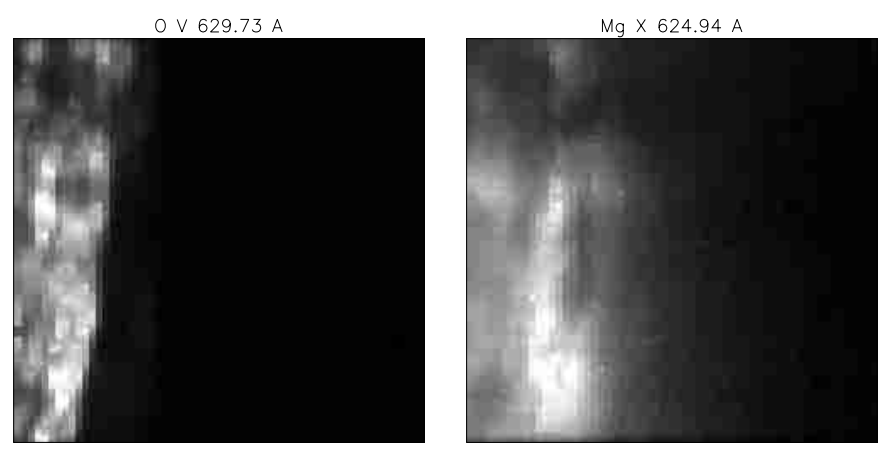

$$
\begin{gathered}
\text { SOHO/CDS NIS Raster, 5-Jul-2001 06:59:47 } \\
\text { WAVE1-- Deep Limb Scan-- s22782ro6.fits } \\
\text { Center }=\left(1015^{\prime \prime},-77^{\prime \prime}\right) \text {, Size }=244^{\prime \prime} \times 240^{\prime \prime}
\end{gathered}
$$
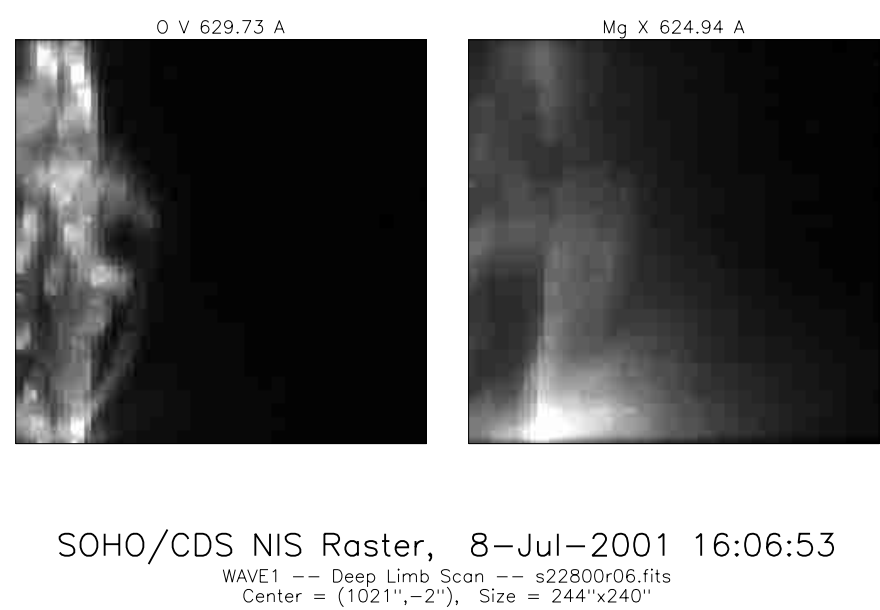

Fig. 1. One rastered image from each of the two observation sequences, for July 5 (top) and July 8 (bottom) 2001. In each case, coincident $4 \times 4$ arcmin square images in the lines from O V $629 \AA$ $(250000 \mathrm{~K})$ and $\mathrm{Mg} \mathrm{X} 624 \AA$ (1 million K) are shown.

The images were centred on 1014 arcsec west of Sun centre, and 77 arcsec south of Sun centre. Thus, we are observing an area just south of the western limb, with most of the image offlimb. Each CDS observation run has an associated run number, which, for reference, in this case is 22782 .

For the July 8 data, observations were made from 12:24 UT and 17 rasters were made, ending at 22:17 UT. In this case, the pointing was 1021 arcsec west and 1.8 arcsec south. Thus, we are observing effectively on the equator. The CDS run number is 22800 .

The principal aim of this study is to identify the relationship, if any, between line-width, altitude and intensity. The greatest problem in such a venture is the low intensity of offlimb spectral lines. However, the innovation enabled by this observation is based on the fact that the limb is effectively running north-south and we can improve the statistical significance of the intensities by accumulating line intensities over time and latitude.

Of the 12 rasters from the July 5 run, two have pixel groups with data-gaps. We ignore these two and sum the other 10 rasters. This assumes that the Sun is rather inactive during the observation sequence, i.e. that there are no significant changes with time. However, we are viewing a quiet, "diffuse" coronal region, so this is a fair procedure. We are left with one image array of $60 \times 143$ pixels. In the east-west direction, we have 60 positions of size 4 arcsec, and in the north-south direction, 143 pixels of size 1.68 arcsec. To ensure good counting statistics, we also sum pixels in the north-south direction. However, inspection of Fig. 1 shows that there is a bright coronal region in the southern portion of the image. Our aim is to investigate the quiet Sun corona, so we eliminate this region by summing the upper 83 pixels in the north-south direction (60 to 142). Again, this is assuming that the physical nature of the corona does not vary significantly over that region. The rastered images were made sufficiently close to the equator, over a quiet Sun region to ensure that the remaining 60 pixel array provides a good measure of intensity versus altitude for each of the 4 selected lines. In addition, for each of the pixels we have 40 pixels in the wavelength dimension.

Using this method we have established a data-set with good statistical significance, for exploring the quiet Sun line-width relationship to intensity and altitude.

Several instrumental corrections have to be applied. For the original images we have run standard routines to clean images of cosmic ray hits, which produce occasional anomalously bright pixels. In general these are easily identified due to the extreme, compact, transient nature of each cosmic ray associated brightening. Given the nature of the CDS CCD detector system (see Harrison et al. 1995) we apply a debias procedure to the images to ensure that the read-out electronics does not produce an artificial bias to the count-rates from different read-out quadrants of the detector. Again, this is a straightforward procedure. We do not apply a final calibration routine (to convert from counts to photons per second); the data are left as counts because the current study is looking for trends rather than absolute values in the line intensities.

Similarly, for the July 8 data-set, we identified one image with a data-gap and summed the remaining 16 as described for the July 5 observation. Again, there is a bright region in the corona in the lower portion of the image. It appears to be a bit more extended that for July 5 , so we sum the upper 43 rows (100 to 142$)$.

Figure 2 shows the plot of line intensity versus pixel number for the $\mathrm{O} \mathrm{V}$ and $\mathrm{Mg} \mathrm{X}$ data for the July 5 observation. The geometry of the observation is such that we can effectively translate the pixel number, or bin, directly into altitude. Each bin is $4 \operatorname{arcsec}$ or $3000 \mathrm{~km}$ in size. The body of the Sun is to the left and the location of the limb is clearly identified by the $\mathrm{O} \mathrm{V}$ drop between bins (pixels) 12 to 14 . We define bin 13 as the limb, and, noting that we are detecting emission over 60 bins, we are recording to an altitude of $140000 \mathrm{~km}$ above the limb.

The line intensities are produced by fitting to the emission line data for each pixel, removing background and summing the counts within the emission line only. The fitting procedure used is a facility within the Atomic Data and Analysis Structure (ADAS) codes (Summers 1994). The CDS emission lines are not perfect Gaussian shapes but have "raised wings". The ADAS procedures contain the best line fit parameters for the CDS emission lines and these are used to make the fits used in this work. The intensities are given in counts. Given the 

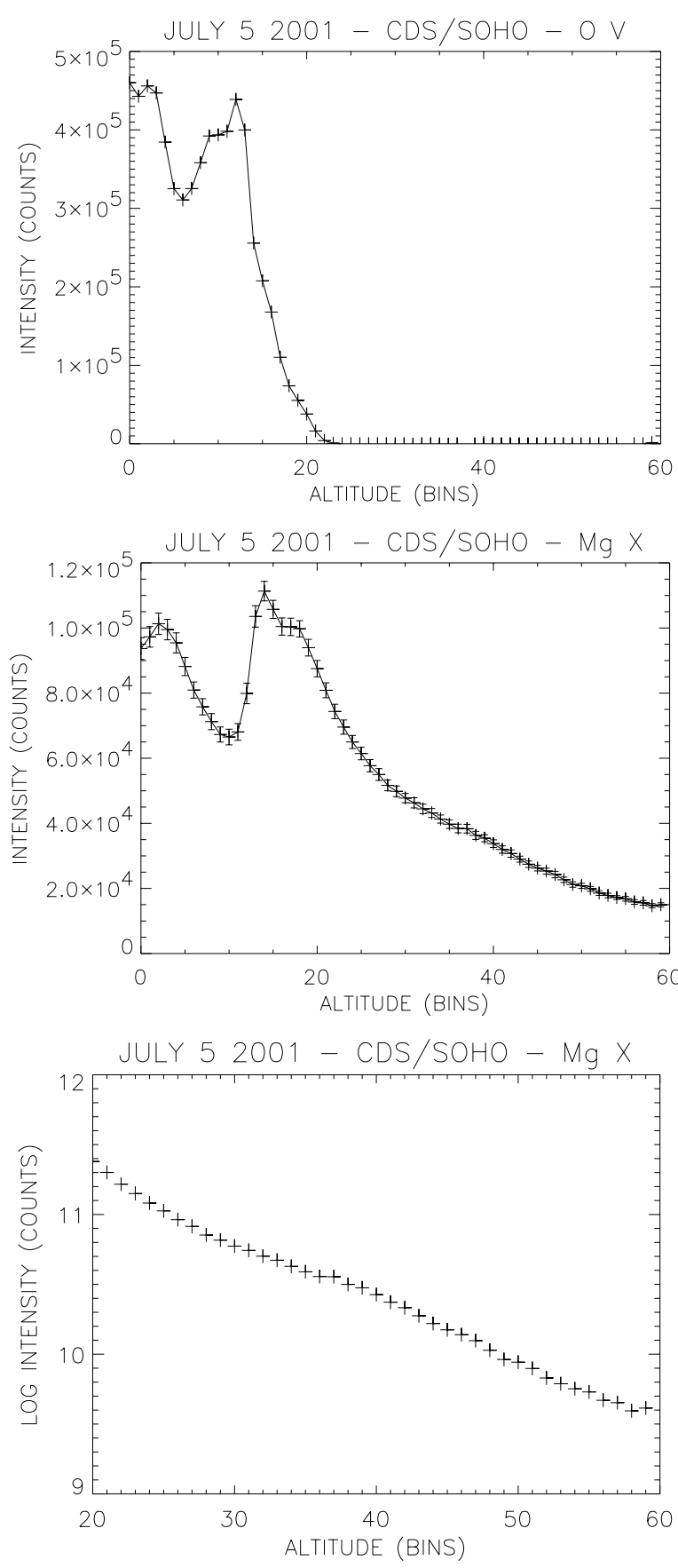

Fig. 2. The altitude-intensity profiles for the $\mathrm{O} \mathrm{V}$ and $\mathrm{Mg} \mathrm{X}$ emission lines for the July 5 observation.

statistical error on the line intensities of the pixels we can also assign an error to the fitted line-width. It should be mentioned that the emission line shapes recorded by the CDS NIS instrument showed a change after the loss and subsequent recovery of SOHO in 1998. The post-recovery line shapes have enhanced wings relative to the pre-loss data. This is catered for in the current fitting routines.

Figure 2 shows that there is some weak $\mathrm{O} \mathrm{V}$ emission, possibly from low-lying loops up to bin 21 but from that point the $\mathrm{O} \mathrm{V}$ intensity is effectively zero. The $\mathrm{Mg} \mathrm{X}$ intensity curve shows limb brightening and a peak right on the limb. This effect is clearly seen in Fig. 1 and is simply due to the line of sight integration through the hot low coronal plasma. From this point the intensity falls with altitude, but shows some brightening in the low corona (bins around 16-19). This appears to coincide with a wing of enhanced emission in the $\mathrm{O} V$ data up to bin 21 , after the initial precipitous fall in intensity. However, for the $\mathrm{Mg}$ X data, intensities of approximately 20000 counts are recorded even at the hightest altitude, due to the pixel summing procedures. The error bars are shown, and this stresses the statistical significance of the data-set.

We are investigating the line-width trends with intensity and altitude for the "clean" quiet Sun. Thus, to exclude any influence of low-lying loops and the limb, we must concentrate on the altitude bins 20 to 60 . For this region, we plot, in the lowest panel of Fig. 2, the natural $\log$ of the intensity against altitude.

This plot shows a drop of a factor $e$ over approximately $69000 \mathrm{~km}$ in altitude. Given hydrostatic equilibrium, we would expect density, $n$, to fall with the function,

$n=n_{0} \times \mathrm{e}^{-\left(r-r_{0}\right) / H}$

where $H$ is the density scale-height. The EUV emission is due to collisional processes, i.e. intensity $(I)$ is proportional to the square of the density. Thus,

$I=I_{0} \times \mathrm{e}^{-2\left(r-r_{0}\right) / H}$.

Thus, the intensity scale-height of $69000 \mathrm{~km}$ translates to a density scale-height of $34500 \mathrm{~km}$.

The theoretical scale-height can be calculated from $k T / m g$ over small relative distances, where $m$ is the proton mass, $k$ is Botlzmann's constant, $T$ is the temperature and $g$ is the acceleration due to solar gravity in the corona. For the one million $\mathrm{K} \mathrm{Mg} \mathrm{X} \mathrm{plasma,} \mathrm{this} \mathrm{gives} 30000 \mathrm{~km}$, which is remarkably similar to the measured value of $34500 \mathrm{~km}$. Thus, despite the inevitable presence of the magnetic structure, it seems that coronal magnetic activity and plasma dynamic processes are not restricting gravitational settling across the observation.

Figure 3 shows the $\mathrm{Mg}$ X line-width (half 1/e width) versus altitude and intensity, for the July 5 observation.

Before proceeding, let us examine the expected line width value. Solar emission line widths have three basic components, namely, (i) an instrumental width, which is defined by the width of the image of the slit on the detector plane, (ii) a thermal width which is the natural width of the emission line due to the temperature of the emitting plasma, and (iii) a nonthermal width, i.e. broadening which may be due to turbulence or plasma motion. For an instrument like CDS, the instrumental width dominates the emission line width, but it is a known component and it does not change with time.

We may write the width of an emission line detected using CDS as,

$\frac{\Delta \lambda}{\lambda} \propto\left(\frac{k T}{M_{i}}+\phi+\xi\right)^{1 / 2}$

(see McClements et al. 1991), where the three terms are the thermal width, the non-thermal width and the instrumental width, from left to right. We are making the assumption in this study that the ion and electron temperatures are identical, 


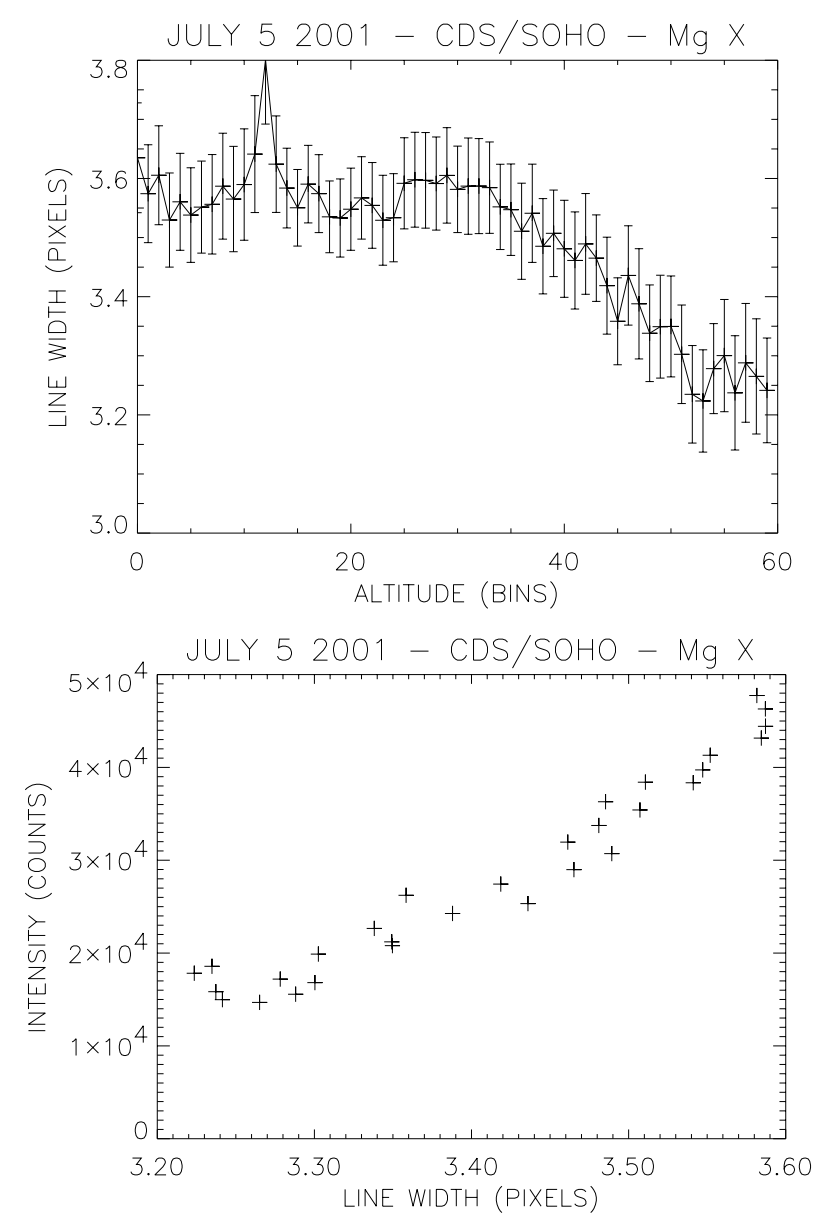

Fig. 3. Plots of the July $5 \mathrm{Mg} X$ line-width (half $1 / e$ width) versus altitude, and line-width versus intensity. The latter is for data above altitude bin 35 .

for the height range under discussion. We believe this to be a reasonable assumption.

We note that each CDS pixel for the band containing the $\mathrm{Mg}$ X line is of size $0.14 \AA$ (see Harrison et al. 1995). Thus, for the instrumental component, $\xi$, given by the width of the image of the slit on the detector plane, we anticipate the contribution from this component to be of order $0.28 \AA$ (see Harrison et al. 1995 ) since we are using a $4 \operatorname{arcsec}$ slit. This represents a $\Delta \lambda / \lambda$ of 0.00045 at $625 \AA$.

For a million K plasma with an emission line at $625 \AA$, i.e. the $\mathrm{Mg} \mathrm{X}$ observation, the thermal contribution is $0.077 \AA$, i.e. a $\Delta \lambda / \lambda$ contribution of 0.000124 .

Again, we stress that the instrumental width dominates. In fact, CDS was not designed with emission line profile analyses in mind. However, the fact that the instrumental (and thermal) components to the line-width are known means that we can explore the physics of line-width changes with this instrument, but, given a good line selection, any change suggests a change in the non-thermal component.

The non-thermal contribution to EUV emission lines is often considered to be about equal to the thermal contribution. This being the case, the total line width anticipated would be of order $0.30 \AA$ (i.e. the square root of $0.28^{2}+0.077^{2}+0.077^{2}$ ). This amounts to 2.14 pixels. This is an estimation of the full width half maximum value of the emission line. The analyses for the figures given in this paper make use of the 1/e width, which will be larger. Nevertheless, the lines are a little wider than anticipated and this is consistent with figures noted by Lang et al. (2000) for pre-launch line-width measurements. It is thought that there is slight defocus in the CDS instrument such that the line width is larger than expected for an instrument with perfect alignment. Again, it should be stressed that the CDS instrument is designed to measure and compare line intensities rather than line profiles. The measurement of line shapes and widths is possible but must be taken with care.

The half width at the $1 / \mathrm{e}$ intensity is noted for each line fit, and this is plotted against altitude (bin) and intensity, in Fig. 3, for the Mg X observation of July 5. We calculate error bars for the line width and this stresses, again, that the pixel summing technique has provided statistically significant results. It is these error bars (in line width) that we have attempted to bring down to manageable levels using the temporal and pixel summing.

Given the error bars, the $\mathrm{Mg} \mathrm{X}$ line-width shows effectively no variation on the solar disc, across the limb and the low corona, except for a peak exactly on the limb. The width stays at values near 3.5-3.6 pixels. However, from altitude bin 30 we see a significant change, with clear line-width narrowing as a function of altitude from about 3.55 to 3.25 . This is a line narrowing of almost $10 \%$ in the altitude range 50000 to $140000 \mathrm{~km}$, or a gradient of -0.01 pixels per altitude bin. However, below $50000 \mathrm{~km}$ altitude, the line width is apparently constant, except for the limb itself. We believe that the presence of low-lying loops (see comments above), in pixels up to number 21, means that we cannot consider the off-limb corona below this altitude to provide a useful measure of the quiet coronal line width.

We note that the on-disc data here are for quiet Sun. Hassler et al. (1990) reported off-disc widths greater than the on-disc widths but his may be due to solar activity. For comparison, the Doyle et al. (1998) study covered the altitudes to only bin 22 in Fig. 3. Although there is a suggestion of broadening in bins 14 to 17 , as well as bins 22 to 32, which would be consistent with the findings of Doyle et al., it is a marginal effect. The Hassler et al. broadening was detected to the equivalent of altitude bin 36. Again, we see marginal broadening effects, but the principal finding of narrowing is at greater altitudes.

Also, in Fig. 3 we plot the line-width against intensity. We only plot this for intensities below $5 \times 10^{4}$ counts. This is the region of the narrowing of the $\mathrm{Mg} \mathrm{X}$ emission line, above altitudes of $50000 \mathrm{~km}$. We find a positive relationship between intensity and line-width, with a slight increase in gradient with intensity, as would be expected from the data in Figs. 2 and 3 .

We now turn to the July 8 data-set.

Figure 4 shows the plot of line intensity versus pixel number for the $\mathrm{O} \mathrm{V}$ and $\mathrm{Mg} \mathrm{X}$ data for the July 8 observation. As with the July 5 observation, the geometry is such that we can effectively translate the pixel number, or bin, directly into altitude, and each bin is 4 arcsec or $3000 \mathrm{~km}$ in size. In this case, the $\mathrm{O} \mathrm{V}$ data show the location of the limb to be between bins (pixels) 10 to 12 . For this analysis, we define bin 11 as the limb. 

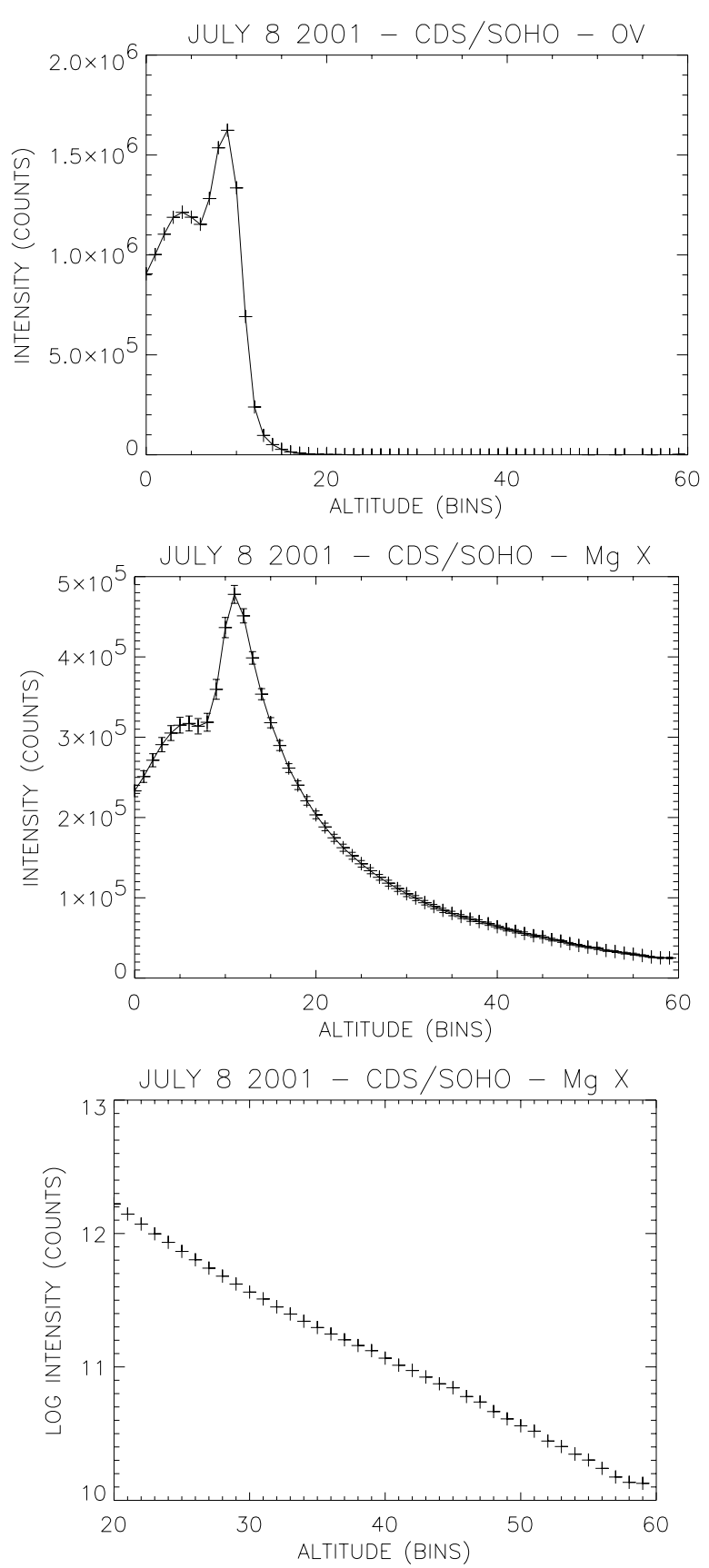

Fig. 4. The altitude-intensity profiles for the $\mathrm{O} V$ and $\mathrm{Mg} \mathrm{X}$ emission lines for the July 8 observation.

The $\mathrm{O} \mathrm{V}$ data also shows significant intensities above the limb, to bin 15.

The $\mathrm{Mg}$ X intensity profile peaks in bin 11, just on the limb. This is consistent with the July 5 observation. The subsequent fall in intensity with altitude is smooth, following an apparently exponential profile.

These intensity-altitude plots suggest that we have "clean", quiet Sun corona from about altitude bin 20 outwards. Again, for this region, in Fig. 4, we plot the natural log of the intensity against altitude. This plot shows an intensity drop of factor $e$ over approximately $60000 \mathrm{~km}$, which according to arguments given above, translates to a density scale-height of $30000 \mathrm{~km}$. This is identical to the expected density scale-height in a
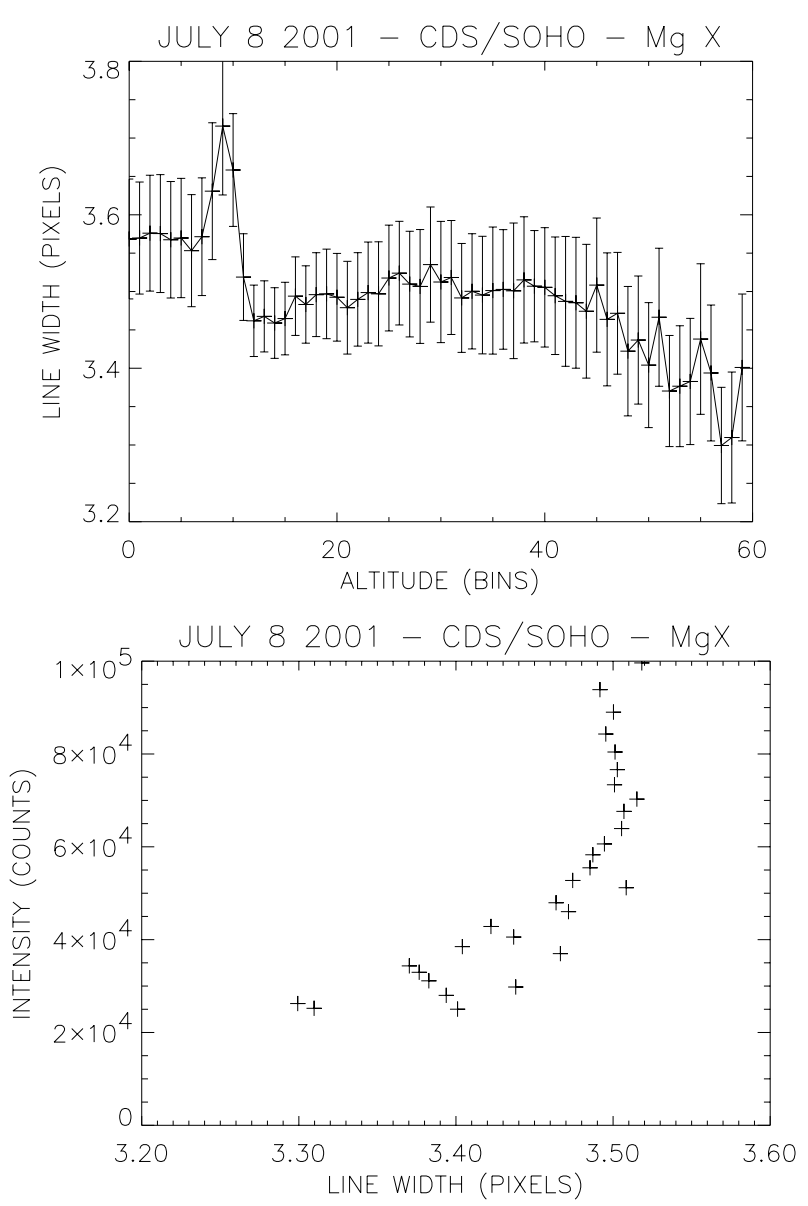

Fig. 5. Plots of the July $8 \mathrm{Mg} \mathrm{X}$ line width (half 1/e width) versus altitude, and line width versus intensity.

million $\mathrm{K}$ corona and suggests that the corona is inactive and that the magnetic fields are allowing settling to occur "naturally".

Figure 5 shows the Mg X July 8 line-width as a function of altitude. The on-disc line-width is constant at about 3.55 pixels. A clear peak in the line-width at 3.7 pixels is identified on the limb, after which there is a decrease in the line width to values less than those on the disc. Our principal interest is the altitudes between bins 20 to 60 and in this region we see an effectively constant line width at 3.5 pixels to bin 40 after which there is a steady decrease of about 0.01 pixels per altitude bin, which is identical to the gradient of the July 5 observation.

We note the contrast to the Hassler et al. (1990) observation where the on-disc line-width was narrower than the offdisc data. Also, again, we point out that the Doyle et al. (1998) and Hassler et al. (1990) observations saw broadening to altitudes equivalent to bins 20 and 34 . Our principal feature, the emission line narrowing, is at a greater altitude.

Also, in Fig. 5, we show the intensity line-width relationship for intensities less than $1 \times 10^{5}$, i.e. for altitudes greater than $60000 \mathrm{~km}$. This shows a similar trend to the July 5 plot, with a positive relationship and evidence for an increasing gradient with intensity.

Finally, we note that the Banerjee et al. (1998) coronal hole observation showed apparent broadening to an altitude of 
$110000 \mathrm{~km}$. This is equivalent to altitude bins 49 and 47 for the July 5 and July 8 data, respectively. This is well into the altitude range for which we detect narrowing but the difference may be due to the fundamental difference of the coronal hole (open field) and equatorial (closed field) coronae.

\section{Scattered light}

The CDS telescope delivers an image of the Sun to the entrance slit of the NIS. The slit allows an image of a selected portion of the Sun into the spectrometer. However, surface roughness on the CDS telescope and scan mirror system must produce some degree of scatter from the solar image outside the region imaged by the slit, into the spectrometer. This is particularly important when making observations off the solar disc because the scattered light from the near-by, bright solar disc can become very significant relative to the intensity of the off-limb corona.

Off-limb scatter for CDS has been discussed by David et al. (1997). They used the measured point spread function of the CDS telescope (Harrison et al. 1995) and reconstructed above limb intensities for different disc intensity profiles. These were compared to measured intensities for O III and O VI emission lines, with some success. As an example, they demonstrated that for the O III emission line at $703 \AA$ the scattered light intensity falls to below $10^{-3}$ of the disc intensity over the first 0.04 solar radii or $28000 \mathrm{~km}$ ( 9 bins in altitude of Figs. 2 or 4). Note that this is for a case where the disc is bright, i.e. transition region rather than coronal emission. For the same data and calculation, the scattered light intensity level fell to $3 \times 10^{-4}$ and $10^{-4}$ of the disc intensity at altitudes of 0.1 and 0.2 solar radii ( 23 and 46 bins above the limb). For comparison, the altitude ranges of Figs. 2 and 4 are $0-140000 \mathrm{~km}$ and $0-150000 \mathrm{~km}$, respectively, i.e. to about 0.2 solar radii.

The O V intensity profiles of Figs. 2 and 4 are consistent with the values of David et al. and are from similar temperature plasmas. The intensities above 0.03 solar radii are effectively zero, given the exposure times and statistical error of these data.

The $\mathrm{Mg} \mathrm{X}$ data can be considered as a different scattering scenario (case $\mathrm{C}$, as modelled by David et al.) because the disc is not uniformly bright, and it could be argued that the off limb regions will have an even smaller scatter contribution from the disc. This stresses the significance of the Mg X intensityaltitude profiles of Figs. 2 and 4, which fall by only an order of magnitude over the entire region under investigation. The O III modelled and measured fall off was four orders of magnitude in the same range. The contribution due to scattered light must be small in this case, for the altitudes under discussion here. If a simple correlation is made, only $0.2 \%$ of the $\mathrm{Mg} \mathrm{X}$ intensity at the highest altitudes of Figs. 2 and 4 is due to scatter from the disc. For this reason, we do not consider the scattered light to be of significant concern for the current analysis.

\section{Summary}

We summarise the important aspects of the Mg X $625 \AA$ analysis of the July 5 and 8 observations here.
1. We have examined the Mg X $625 \AA$ (million K) linewidth as a function of altitude and intensity for two quiet Sun, off-limb observations. Our analysis indicates that we can measure the $\mathrm{Mg} \mathrm{X}$ line width to 0.1 pixel or less, that is, to better than $3 \%$ of the line width.

2. We find clear evidence in both data-sets for line-width narrowing as a function of altitude above the limb, and there is no evidence of broadening with altitude.

3. For the two observations, the line-width was examined for quiet Sun corona, well off the disc and above any low-lying loop systems. In this "clean" corona, for the July 5 observation we find a clear fall off in line-width from about 3.55 pixels to under 3.22 pixels between $50000 \mathrm{~km}$ and $140000 \mathrm{~km}$ above the photosphere. Similarly, we examine the "clean" off-limb corona for the July 8 observation. In this case, we see an initial apparently constant line width and then a fall off from 3.5 to 3.3 pixels between altitudes of $87000 \mathrm{~km}$ and $150000 \mathrm{~km}$.

4. The July 5 and July 8 observations show on-disc linewidths of about 3.55 pixels, which remain constant on the disc and low corona (to altitude $50000 \mathrm{~km}$ ) for the July 5 data, and show a fall to 3.5 for the July 8 data. Above $50000 \mathrm{~km}$ for the July 5 data, and $87000 \mathrm{~km}$ for the July 8 data, in the "clean" quiet corona, we witness the line narrowing with altitude.

5. Both data-sets show a peak in the $\mathrm{Mg} \mathrm{X}$ line-width for a narrow region exactly on the limb. This in itself is a surprising result and begs further study.

6. In the "clean" corona, as defined above, both the July 5 and July 8 observation show a positive relationship between line-width and line-intensity.

7. Both observations show line-width narrowing gradients with altitude at 0.01 pixels per altitude bin, which is $4.7 \times$ $10^{-6} \AA / \mathrm{km}$.

8. Past data have shown evidence for broadening with altitude, to altitudes of $25000 \mathrm{~km}$ and $70000 \mathrm{~km}$. Evidence for broadening to such altitudes in the current data-set to these altitudes is, at best, marginal and this may be due to differing low-coronal structures for the different data-sets. However, the past data-sets were not able to identify the narrowing which was detected at higher altitudes. One polar coronal hole observation made over a similar altitude range did not detect narrowing at high altitudes but this is interpreted as being due to the fundamental difference between the open and closed field regions of the coronal hole and equatorial corona. In short, the key here is the fact that the current study is an observation of an extremely quiet equatorial corona.

9. For both data-sets the apparent density scale-heights appear to match calculated scale-heights for a million K plasma in the solar atmosphere, suggesting that the corona is inactive and in or near a hydrostatic equilibrium condition.

The most important result here is that there is strong evidence for an inverse relationship between line-width and altitude, for the quiet corona, for altitudes over $50000 \mathrm{~km}$. This, we believe is the first such observation of emission line narrowing in the off-limb corona. However, another important result to explain is the narrow region of broadening of the $\mathrm{Mg} \mathrm{X}$ emission line actually on the limb. 


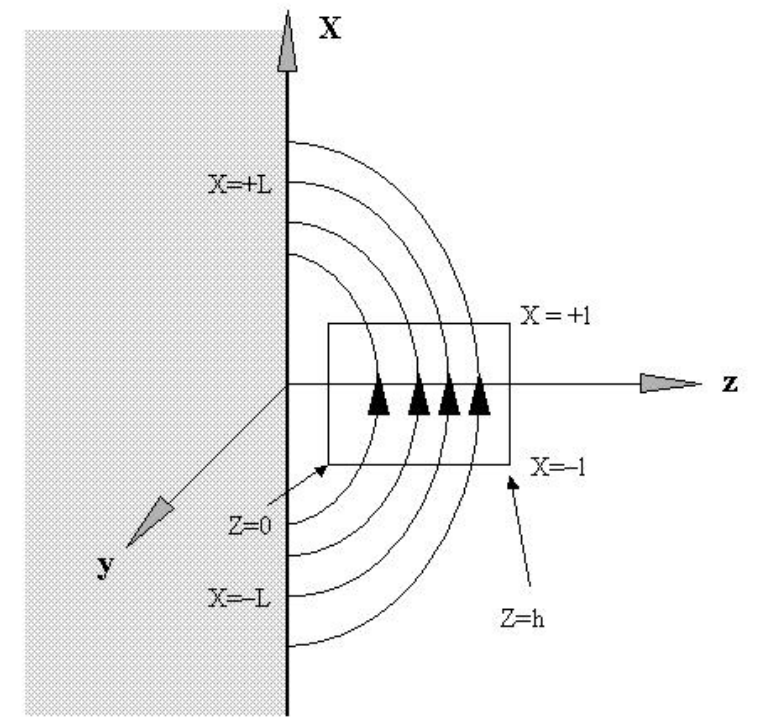

Fig. 6. A schematic of the observation used to model the broadening results (see text).

\section{Theoretical interpretation}

There are many possible explanations of line width broadenings, including turbulence and other unresolved velocities. However, a simple model that is consistent with these observations is that of damped Alfvén waves. This section presents a simple model to illustrate the ideas and is a starting point for subsequent more detailed modelling.

Consider a basic magnetic configuration shown in Fig. 6, with large-scale closed loop structures spanning the equator. Although we are not able to resolve these loops, in the quiet solar equatorial regions, this is the most sensible scenario to assume. We are not observing a coronal hole; there is diffuse emission above the limb, which presumably denotes the presence of closed loops. Alfvén waves may be excited at the footpoints by lateral motion of the field lines. If, for a particular field line, the amplitude of the motion is given by $V_{y}$, then the energy flux, $F$, propagating into the corona along the field lines can be given as

$F=C \frac{1}{2} \rho_{0} V_{y}^{2} V_{\mathrm{A}}$

where the terms on the right hand side of the equation are a constant, the mass density, the transverse velocity amplitude and the Alfvén velocity, which is given by

$V_{\mathrm{A}}=\frac{B(x, z)}{\sqrt{\mu \rho_{0}(x, z)}} \cdot$

The past studies due to Hassler, Doyle, Banerjee and coworkers, point to the fact that for a constant value of $F$, propagating into regions of decreasing density and magnetic field, the value $V_{y}$ must increase. However, in this study we witness a narrowing of the line as a function of altitude in the corona, which suggests a decrease in $V_{y}$. This, then suggests that the energy flux is decreasing - i.e. there is wave dissipation.

The boxed area of Fig. 6, bound by $Z=0$, h and $X=-1$, +1 represents the area of interest in the CDS image. A simple reason why the velocity amplitudes associated with the line broadenings may be decreasing with height is that the higher altitude magnetic field lines in the boxed area are longer than the lower ones. If the coronal magnetic field lines are oscillated at the photospheric base harmonically with a fixed frequency $\omega$, then the Alfvén velocity amplitude will approximately satisfy an equation of the form

$-\omega^{2} v_{y}=V_{\mathrm{A}}^{2} \frac{\mathrm{d}^{2} v_{y}}{\mathrm{~d} s^{2}}-i \omega \eta \frac{\mathrm{d}^{2} v_{y}}{\mathrm{~d} s^{2}}$,

where $s$ is the distance along the field lines from the summit towards the photospheric ends at $s= \pm L$. The damping is due to the magnetic resistivity, $\eta$, but the same argument holds also for damping due to viscosity. Since we have assumed a harmonic time dependence, the photospheric boundary conditions will be $v_{y}(L)=1$ with a symmetry condition at $s=0$. This simple equation ignores the effects of curvature and any coupling to fast magnetoacoustic waves but will demonstrate how the amplitude of the disturbances can decrease with height if the driving frequency is not a normal mode of the loop.

Assume that the Alfvén speed, $V_{\mathrm{A}}$, is uniform. The solution is given by

$v_{y}(z)=C \mathrm{e}^{i k s}+D \mathrm{e}^{-i k s}$,

where $C$ and $D$ are constants and

$k^{2}=\frac{\omega^{2}}{V_{\mathrm{A}}^{2}-i \omega \eta}$.

$k$ can be expressed as a real part, $k_{0} \approx \omega / V_{\mathrm{A}}$, and an imaginary part $\epsilon \approx \omega^{2} \eta / 2 V_{\mathrm{A}}^{3}$. In order to satisfy the symmetry condition, $D=C$, and the photospheric boundary condition gives

$1=C\left(\cos k_{0} L \cosh \epsilon L-i \sin k_{0} L \sinh \epsilon L\right)$.

Thus, the amplitude of the velocity, $V$, at the mid-point, $s=0$, varies with height as

$v_{y}=\frac{1}{\cos ^{2} k_{0} L+\sinh ^{2} \epsilon L}$.

Thus, if $k_{0} L \neq(n+1 / 2) \pi$, namely the driving frequency is not a normal mode of the field lines, then the amplitude will decay exponentially for large $L$. This means that the line broadening will also decrease with height. The height of the field lines at the summit, $Z$, is related to the length by $Z=L / \pi$ for approximately semi-circular field lines. The variation of the line broadening can be inspected by plotting the variation of the square of the velocity with height. For an Alfvén speed of $2 \times 10^{6} \mathrm{~m} \mathrm{~s}^{-1}$ and a period of $300 \mathrm{~s}$, we obtain $k_{0}=0.01 \mathrm{Mm}^{-1}$. As an example, we consider $\epsilon=0.01 \mathrm{Mm}^{-1}$ as well. This is shown in Fig. 7.

While the general trends are consistent with the observations presented in Figs. 3 and 5, the value of the dissipation coefficient used is much larger than the value predicted from classical values.

An alternative mechanism for the damping of the waves with height is due to phase mixing (Heyvaerts \& Priest 1983; Hood et al. 1997). Waves propagating in the North-South direction, from the photospheric footpoints will encounter a density that decays with height above the solar limb. However, from 


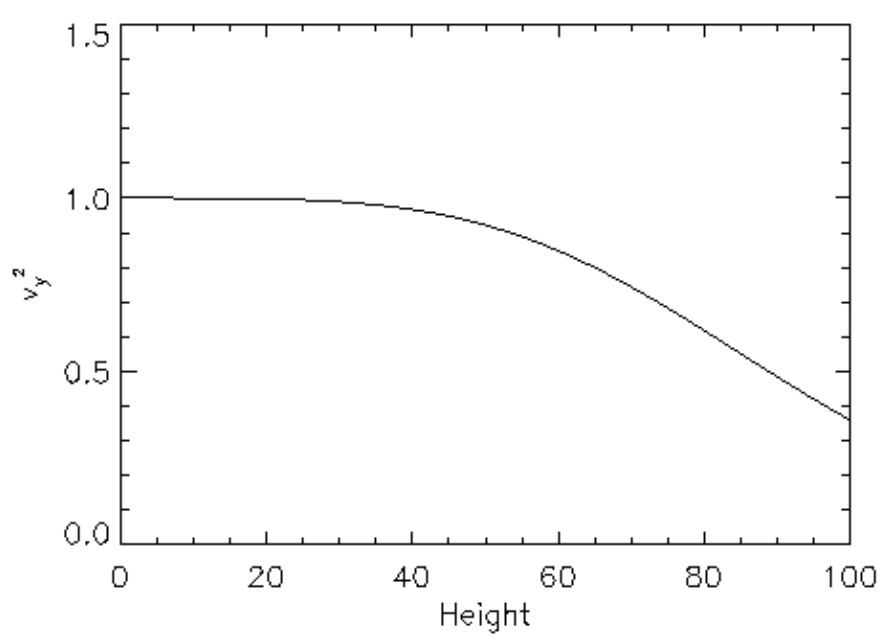

Fig. 7. The variation of the square of the velocity (arbitrary units) with height measured in Mm. As an example, we take $k_{0}=0.01 \mathrm{Mm}^{-1}$ and $\epsilon=0.01 \mathrm{Mm}^{-1}$.

the results of Hood et al. (1997), the Alfvén speed must decrease with height in order to obtain a velocity amplitude decay with height. Thus, the magnetic field strength must initially decrease with height faster than the density decreases due to gravity. This is the case for a dipole field. Alternatively, the waves may convert into fast magnetoacoustic waves that can propagate across the field lines and leave the system.

A more detailed theoretical explanation of the decrease in line broadening with height will require more realistic modelling and is beyond the scope of this paper. The main purpose of this section is to show that the decrease of line broadening with height is consistent with our theoretical understanding of Alfvén wave propagation in the solar corona.

Our principal aim has been to identify the relationship between altitude and line widths, and to explore the possible meaning of this relationship in terms of wave activity. However, another important result, coupled with this is the observation of a narrow region of line broadening exactly on the solar limb. We do not address this observation in detail here, but acknowledge that it is highly suggestive of a line of sight effect of plasma flows in low-lying coronal loops in the limb region.

\section{Discussion}

To the authors knowledge, this work shows the first clear observation of emission line narrowing with altitude in the low corona. We believe this to be an extremely important result because it most likely indicates the dissipation of wave activity.

Previous studies have identified line broadening with altitude. These were, in the main, for coronal holes, or for equatorial regions at altitudes lower than those studied here. The coronal hole situation may be very different from the magnetic configurations of the current work, with undamped waves propagating through open structures; our work is considering largescale closed loops. The earlier equatorial studies may not have observed altitudes where the dissipation occurs.

We do note that some hints of emission line narrowing, or at least a leveling off of the width-altitude profile, have been demonstrated by Doyle et al. (1999) and Doschek et al. (2001) for coronal holes, but not with sufficiently low uncertainties to confirm that the narrowing is a real effect. This was only for some specific lines, and in other studies, no departure from the width increase with altitude was found. The only equatorial studies for which we can compare the current results are due to Doyle et al. (1998) and Doschek \& Feldman (2000). The former did not make observations to the altitudes where we recorded line narrowing and the latter did not identify any specific width-altitude profile due to the uncertainties involved.

Our interpretation of waves in a closed loop system appears to demonstrate wave dissipation through damping, perhaps with the inclusion of phase-mixing, for example. Such an observation provides direct evidence for energy dissipation in the corona and it is thus an important result; the relevance of this result to the general coronal heating problem must be investigated thoroughly. Is this an unusual observation, or a common one? Certainly, the result was only obtained after careful summing over time and space to obtain the necessary statistical significance. However, to investigate this result further, we must make many more similar observations, both in closed and open fields, and a more complete theoretical interpretation must be produced. That being said, the observation of narrowing and its significance as a possible signal of wave dissipation is an important result.

Acknowledgements. CDS was built and is operated by a consortium led by the Rutherford Appleton Laboratory and including the Mullard Space Science Laboratory, the NASA Goddard Space Flight Center, Oslo University and the Max-Planck-Institute for Extraterrestrial Physics, Garching. SOHO is a mission of international cooperation between ESA and NASA.

\section{References}

Banerjee, D., Teriaca, L., Doyle, J. G., \& Wilhelm, K. 1998, A\&A, 339, 208

Banerjee, D., Teriaca, L., Doyle, J. G., \& Lemaire, P. 2000, Sol. Phys., 194, 43

David, C., Gabriel, A. H., \& Bely-Dubau, F. 1997, Proc. 5th SOHO Workshop, ESA SP-404, 313

Doschek, G., \& Feldman, U. 2000, ApJ, 529, 599

Doschek, G., Feldman, U., Laming, J. M., Schühle, U., \& Wilhelm, K. 2001, ApJ, 546, 559

Doyle, G. J., Banerjee, D., \& Perez, M. 1998, Sol. Phys., 181, 91

Doyle, G. J., Teriaca, L., \& Banerjee, D. 1999, A\&A, 349, 956

Harrison, R. A., Sawyer, E. C., Carter, M. K., et al. 1995, Sol. Phys., 162,233

Harrison, R. A., \& Lyons, M. 2000, A\&A, 358, 1097

Hassler, D. M., Rottman, G. J., Shoub, E. C., \& Holzer, T. E. 1990, ApJ, 348, L77

Heyvaerts, J., \& Priest, E. R. 1983, A\&A, 117, 220

Hood, A. W., Ireland, J., \& Priest, E. R. 1997, A\&A, 318, 957

Lang, J., Kent, B. J., Breeveld, A. A., et al. 2000, J. Opt. A: Pure Appl. Opt., 2, 88

Mason, H. E., Young, P. R., Pike, C. D., et al. 1997, Sol. Phys., 170, 143

McClements, K. G., Harrison, R. A., \& Alexander, D. 1991, Sol. Phys., 131, 41

Moran, T. G. 2001, A\&A, 374, L9

Summers, H. 1994, Joint European Torus Report, Atomic Data and Analysis Structure, JET-IR (94)-06 